\title{
Secondary Hemophagocytic Lymphohistiocytosis Complicated by a Cavitary Lung Lesion in a Kidney Transplant Recipient
}

\author{
Katarzyna Muras-Szwedziak Maciej Tylski Anna Masajtis-Zagajewska \\ Michał Nowicki \\ Department of Nephrology, Hypertension and Kidney Transplantation, Medical University \\ of Lodz, Lodz, Poland
}

\section{Keywords}

Secondary hemophagocytic lymphohistiocytosis · Pulmonary complication · Cavitary lung lesion · Renal transplant

\begin{abstract}
Hemophagocytic lymphohistiocytosis (HLH) is an uncommon life-threatening condition caused by an uncontrolled immunological response. It can develop secondary to malignancies, infections, systemic diseases, and immunosuppression. Multiple risk factors may present in kidney transplant recipients; however, the cases of HLH in this population have been described sparsely. We report a case of a 39-year-old female kidney transplant recipient who presented to the hospital nearly 3.5 years after the transplantation with general malaise, recent history of weight loss, fevers, and persistent anemia. Laboratory tests showed pancytopenia, hyperferritinemia, hypertriglyceridemia, and increased activity of lactate dehydrogenase. A bone marrow aspiration revealed hemophagocytosis, which led to the diagnosis of $\mathrm{HLH}$. Therapy consisting of high-dose steroids and plasma exchanges was administered, resulting in a significant improvement of blood count parameters and the patient's general condition. While searching for the triggering disease, a single cavitary lesion in the right lung was revealed in a chest radiograph. Computed tomography scan, bronchoscopy, and additional laboratory testing did not reveal a definitive cause of the lesion. We suspect that the lesion may be a consequence of $\mathrm{HLH}$. The patient was disqualified from thoracic surgery due to multiple comorbidities. Even though HLH is a rare condition, it should be taken into consideration in a kidney transplant patient presenting with unspecific symptoms accompanied by a bicytopenia. It has an unpredictable course that often results in serious complications. Thus close follow-up of the patient and a wide array of imaging and laboratory tests remain crucial.
\end{abstract}




\section{Introduction}

Hemophagocytic lymphohistiocytosis (HLH) also known as the hemophagocytic syndrome is a rare, life-threatening condition caused by an ineffective and uncontrolled immunological response $[1,2]$. At the core of $\mathrm{HLH}$, pathophysiology lies a defective cytotoxic function of cytotoxic T lymphocytes and natural killer lymphocytes. Even though immune system reacts to antigens, it may be unable to eliminate them. Additionally, both the modulation and termination of the immunological response in HLH are impaired. This leads to an overproduction of pro-inflammatory cytokines followed by the systemic proliferation of histiocytes $[1,3]$.

HLH can be classified into a primary and a secondary form. The primary HLH is caused by a variety of genetic mutations, which directly affect cytotoxic function of cytotoxic $\mathrm{T}$ lymphocytes and natural killer cells. It typically presents in children. The secondary HLH (sHLH) can occur at any age, with no clear genetic background, usually in presence of conditions or factors that cause either a strong activation of immune system or immunodeficiency $[1,3]$. Although sHLH is considered to be a distinct entity, recent studies show a possible correlation between a primary and secondary form of HLH $[2,4]$. Most common sHLH triggering conditions are, in order, malignancies, systemic infections, and autoimmune diseases [2]. Rarely, it can present in patients receiving a strong immunosuppression, including bone marrow and solid organ transplant recipients.

In the course of hyperinflammation and aggressive histiocyte infiltrates, massive tissue damage and hematological disturbances ensue. Signs and symptoms of HLH are nonspecific, which makes prompt diagnosis difficult even for experienced clinicians [2].

We present the development and clinical course of sHLH in a kidney transplant recipient. The literature on the subject has been scarce [5-8], with the biggest series involving 17 cases of sHLH among a cohort of 4,230 kidney graft recipients [9]. Our case was complicated by the pulmonary lesion different from the typical complications observed in HLH. There have been only singular case reports describing somewhat similar changes in lungs in the course of hyperinflammatory syndromes $[10,11]$.

\section{Case Report}

A 39-year-old female kidney transplant recipient was admitted to our hospital in September 2018 (Fig. 1) due to general malaise, weight loss, fevers up to $38.5^{\circ} \mathrm{C}$, diarrhea, and moderate anemia in follow-up laboratory tests. Her primary renal disease was lupus nephritis with kidney involvement resulting in end-stage renal disease. For this reason, she was on hemodialysis for 3 years while being prepared for the transplantation. The patient received a renal graft from a deceased donor in March 2016. A kidney biopsy was performed in August 2018 , and its histopathological examination showed no signs of either transplant rejection or drug toxicity. High antinuclear antibody count (including elevated anti-double-stranded DNA antibody count) and low C3 and C4 complement components suggested SLE as the cause of the graft failure, despite an ongoing triple immunosuppressive treatment. Aside from that, patient's medical history included the very intensive courses of immunosuppresion including cyclophosphamide, mycophenolate mofetil, cyclosporine A and pulses of intravenous steroids. The patient also suffered from cytomegalovirus (CMV) early after transplantation and a recurrent CMV infection in August 2018.

On the admission, the immunosuppressive regimen consisted of mycophenolate sodium, tacrolimus, and prednisone. The patient was also receiving valganciclovir due to the recent CMV infection. The physical examination showed the signs of dehydration and an enlarged

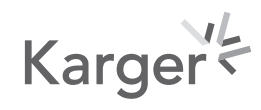




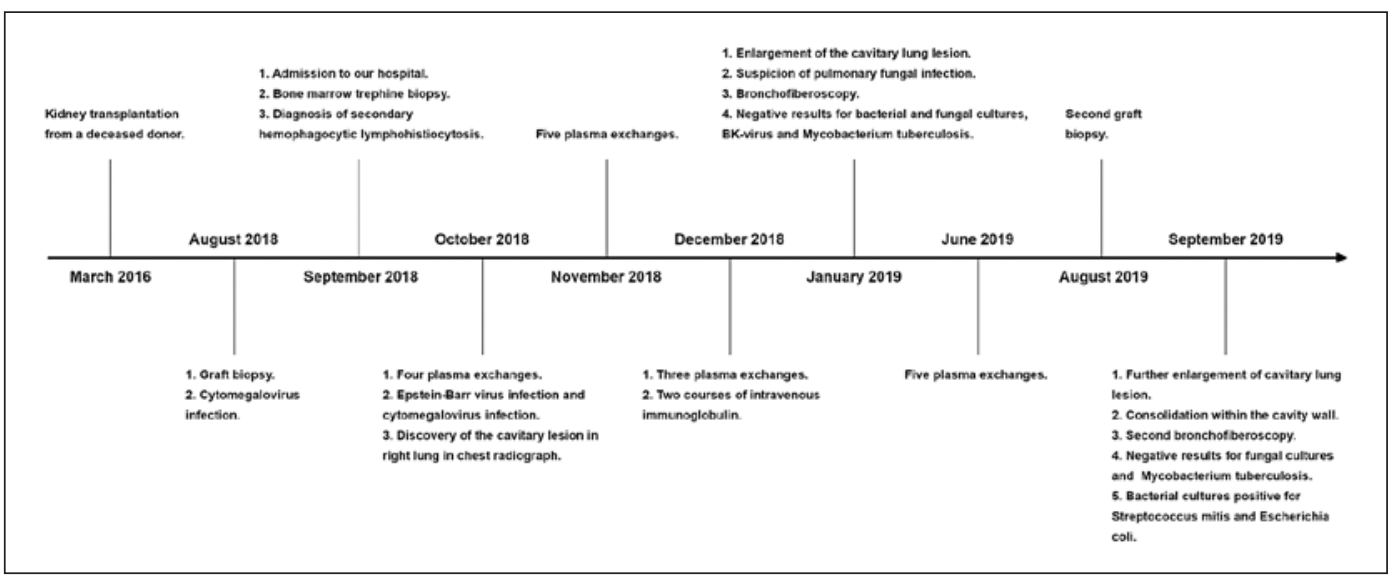

Fig. 1. Sequence of the events.

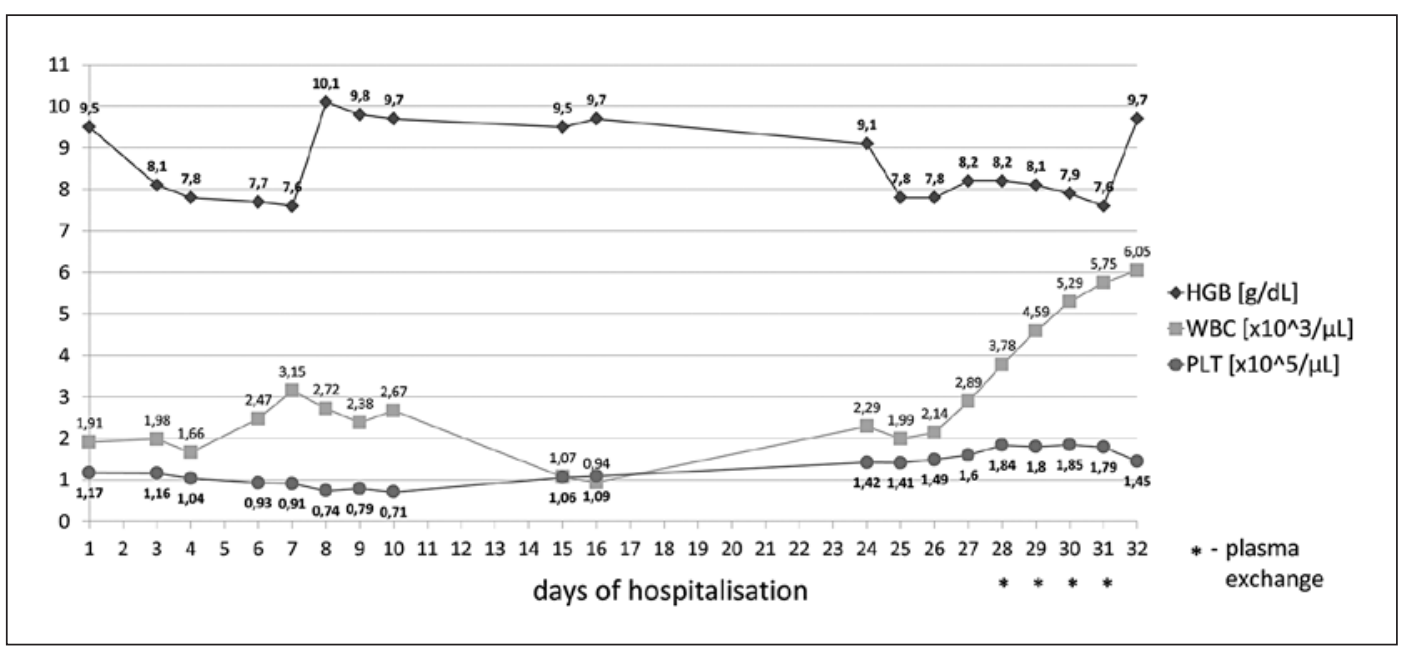

Fig. 2. Changes in blood count from the admission to the first cycle of PE. PE, plasma exchange.

single left submandibular node. The routine laboratory tests revealed the signs of pancytopenia (hemoglobin $9.5 \mathrm{~g} / \mathrm{dL}$; leukocyte count $1.91 \times 10^{3} / \mu \mathrm{L}$; and platelet count $117 \times 10^{3} / \mu \mathrm{L}$ ) and renal failure with metabolic acidosis (serum creatinine $462.5 \mu \mathrm{mol} / \mathrm{L}$; urea $31.9 \mathrm{mmol} / \mathrm{L}$ and $\mathrm{pH}$ 7.153; and $\mathrm{sHCO}_{3}{ }^{-} 11.8 \mathrm{mEq} / \mathrm{L}$ ). Serum C-reactive protein level was within normal range. The abdominal ultrasound was significant only for gallbladder polyps and revealed a single cyst in the upper pole of the transplanted kidney and an accessory spleen. No enlargement of internal organs was seen. The intravenous fluid therapy was promptly started, resulting in a partial improvement of graft function. Since we considered valganciclovir as a potential cause of pancytopenia, CMV-DNA laboratory test was carried out and upon receiving a negative result the drug was discontinued. One unit of leukoreduced and irradiated blood was transfused, and the patient received a single dose of an erythropoiesis-stimulating agent and 2 doses of granulocyte colony-stimulating factor (G-CSF). Despite all this, no significant improvement of blood count was observed (Fig. 2). Among numerous other laboratory tests performed 3 were especially worthy of attention, that is, increased activity of lactate dehydrogenase (LDH) (341 U/L), elevated triglycerides $(2.35 \mathrm{mmol} / \mathrm{L})$, and highly increased serum ferritin $(>2,000 \mathrm{ng} / \mathrm{mL})$. The patient was then transferred to the Hematology Clinic, 


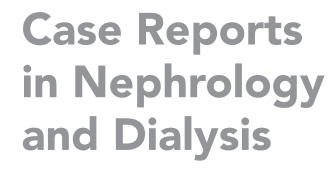

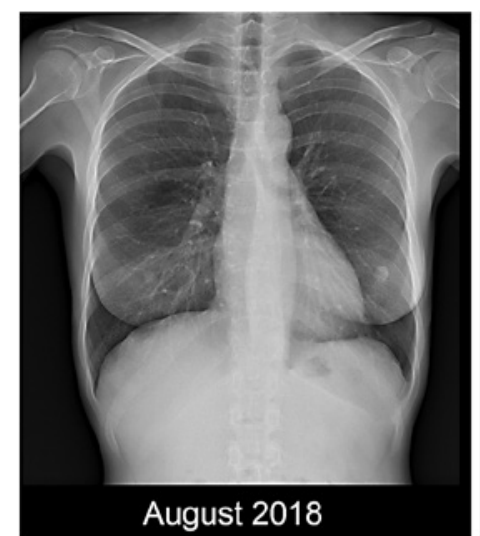
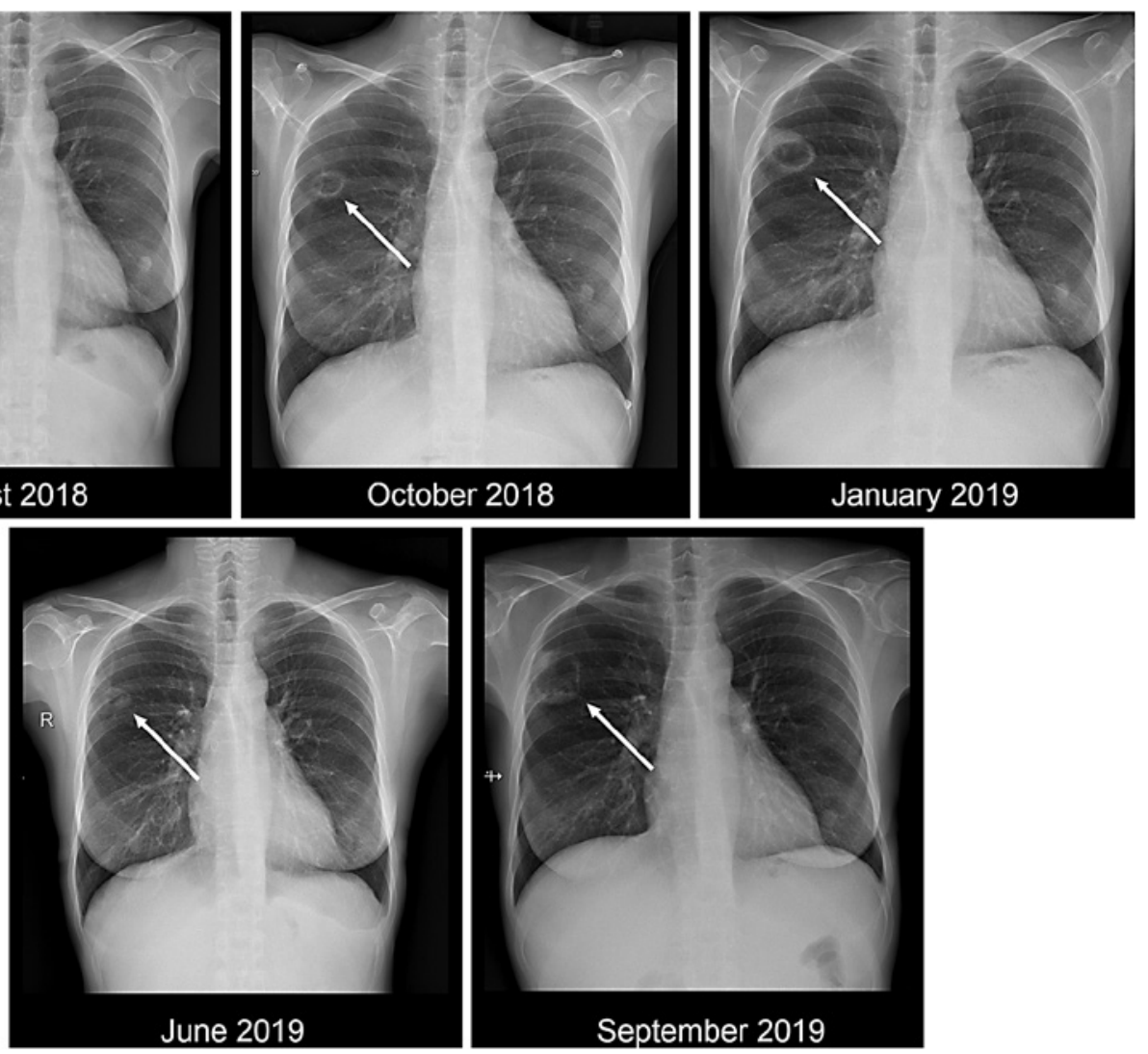

Fig. 3. Chest radiographs in subsequent months.

where she had a bone marrow aspiration performed. The histopathological examination of the bone marrow specimen revealed an intensive hemophagocytosis which alongside the aforementioned laboratory and clinical findings raised a suspicion of sHLH.

After the patient returned to our nephrology ward in October 2018, the possible treatment plans were discussed. Etoposide therapy was considered inordinately toxic given the presence of graft failure and hypoalbuminemia $(25 \mathrm{~g} / \mathrm{L})$. The immunosuppressive regimen was modified, and tacrolimus was converted to everolimus and oral prednisone to an intravenous dexamethasone. Based on the available literature, plasma exchange (PE) therapy was initiated. In total, 4 PEs were performed, resulting in a remarkable improvement of blood count parameters (Fig. 2) and the patient's general condition.

In search for a potential cause of sHLH CMV-DNA and Epstein Barr Virus DNA (EBVDNA), tests were performed. The results of both tests were positive with $1.25 \times 10^{3}$ copies/ mL EBV-DNA and CMV-DNA detectable levels but too low to estimate the viral load. Hence, intravenous ganciclovir was started. The patient was referred to a rheumatologist who suggested a possible SLE exacerbation based on newly presented oral ulcers, right wrist pain, bilateral Reynaud's phenomenon, and previously described signs of renal failure present in laboratory tests. Gastrointestinal malignancies were ruled out after negative results of colonoscopy and gastroscopy. Lastly, a chest radiograph was taken, revealing a single cavitary lesion in the right lung which was not present in the previous radiograph (Fig. 3). Following that, a computed tomography was performed and precise measurements were taken (Fig. 4). According to the radiologist, the observed lesion presented no active inflammation and it was most likely post-inflammatory, although it could also be caused by 
Case Reports in Nephrology and Dialysis

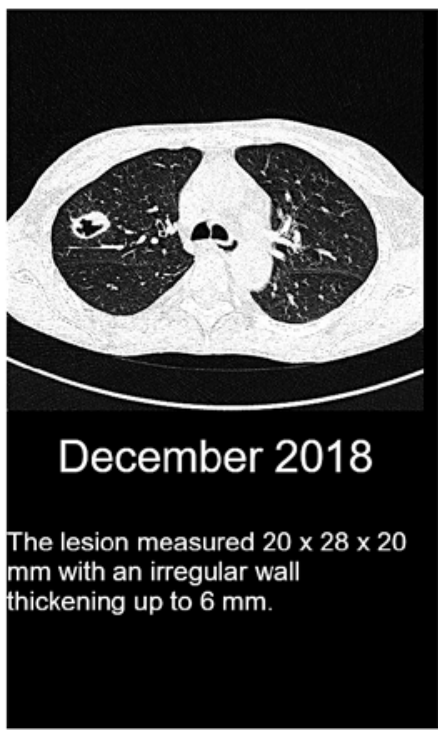

Muras-Szwedziak et al.: sHLH with a Pulmonary Complication in a Kidney Transplant Recipient
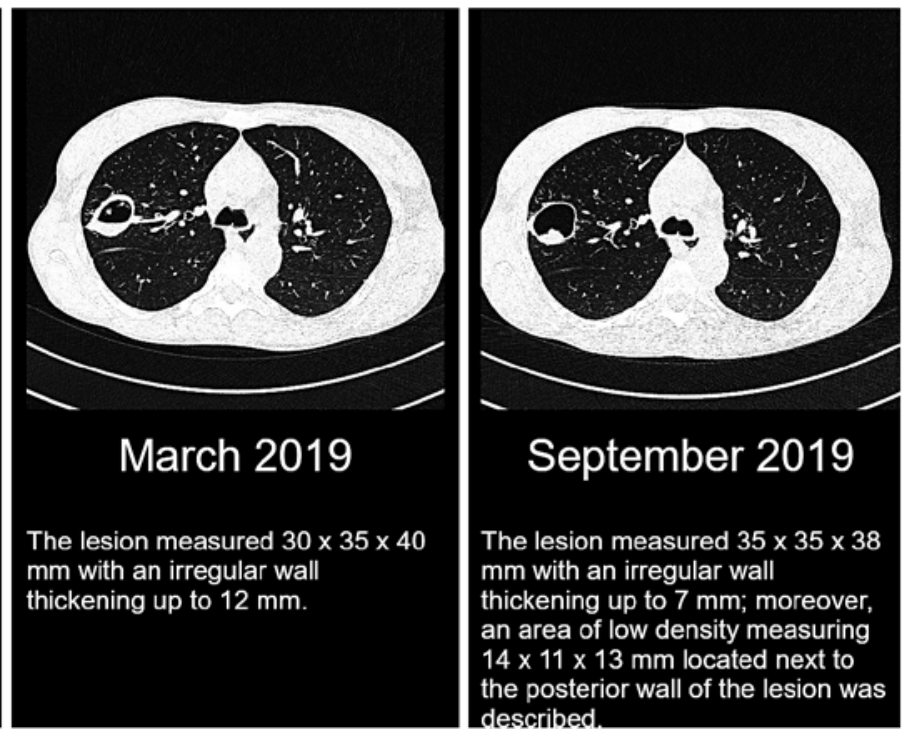

Fig. 4. Computed tomography scans and measurements of the lesion in subsequent months.

tuberculosis. At the moment sustained therapy with reduced doses of immunosuppressants and follow-up of the lesion progression was deemed the reasonable approach. The patient was discharged from the hospital with frequent follow-up visits at our outpatient clinic.

In January 2019, a significant enlargement of the lung lesion was noticed in a chest radiograph (Fig. 3). At this point, a preemptive therapy for the mycobacterium tuberculosis would be useful for the diagnosis; however, it was not administered. Instead, the patient was referred to a pulmonologist, who raised a suspicion of the fungal pulmonary infection. A bronchofiberoscopy was performed, which did not reveal any macroscopic changes. The samples for bacterial and fungal cultures, BK-virus test, Mycobacterium tuberculosis culture, and PCR-DNA were taken. At the same time, blood galactomannan level was also assessed. All these test results came negative. The patient was also referred to a thoracic surgeon who advised an active monitoring of the lesion with no immediate indication for its surgical removal. In the following months, chest radiographs and CT scans showed a slow increase of the size of the cavitary lesion with the thickening of its wall (Fig. 4).

At the same time, the patient suffered from a prolonged course of sHLH. High levels of ferritin, that is, $2,761 \mathrm{ng} / \mathrm{mL}$ in January $2019 ; 1,149 \mathrm{ng} / \mathrm{mL}$ in June 2019; and 1,946 ng/mL in August 2019, and cytopenia affecting from 1 to 3 cell lines were observed during subsequent hospitalizations. The patient had additional courses of PE performed from November 2018 to June 2019-17 in total. Moreover, 2 courses of intravenous immunoglobulin (IVIG) therapy were necessary to maintain disease control. Due to high serum creatinine and urea levels in follow-up laboratory tests, the patient had a second graft biopsy performed in August 2019. The histopathological examination revealed the signs of tubulointerstitial graft rejection and coexisting thrombotic microangiopathy.

In September 2019, a further progression and consolidation in lung CT within the cavity wall was observed; therefore, the patient was referred to a pulmonary disease center. There, a second bronchofiberoscopy was performed which included bronchoalveolar lavage and carinal forceps biopsy. Since changes of the lesion observed in CT were highly suspicious for pulmonary fungal infection, the patient was started on voriconazole. Another set of samples for bacterial, fungal, and Mycobacterium tuberculosis cultures was taken. Cytological examination of the lavage fluid revealed increased amount of neutrophils $(11.3 \%$ of all counted 
leukocytes, with normal range $<3 \%$ ); distribution of the other leukocyte populations, including macrophages, was normal; however, the histopathologist observed signs of activation and phagocytosis in some of the macrophages; additionally, numerous erythrocytes, sparse bacteria, and sparse fungi hyphae were described in the sample. Examination of the specimen from the carinal biopsy showed active inflammation within the mucosa with no fungi hyphae. Bacterial cultures were positive for Escherichia coli and Streptococcus mitis, cultures for Mycobacterium tuberculosis and fungi were once again negative. Antibiotic therapy was administered according to the antibiotic susceptibility testing results.

She was initially qualified to thoracic surgery, but due to severe flu, zoster, and graft failure, the procedure has been postponed. As the graft function gradually worsened, the patient returned to hemodialysis in April 2020.

\section{Discussion}

According to the available literature, sHLH has been most commonly associated with malignancies in about half of the reported cases - followed by the systemic infections and autoimmune diseases [6]. It has been much less prevalent in patients on immunosuppressive therapy $[5,12]$. In a population of kidney transplant recipients, the most commonly identifiable cause of sHLH was viral infections, mainly EBV and CMV [6]. Nearly all of the reported cases were diagnosed in the first few months following the surgery when the most intensive immunosuppressive regimen was required. In comparison, our patient developed SHLH 3.5 years after the surgery. There have been only a few documented cases of post-kidney transplant patient developing sHLH late after the transplantation $[6,8,9]$. Specifying the exact pathophysiology of sHLH in our case poses a great challenge. In light of the mentioned statistical data and given that the patient's EBV-DNA and CMV-DNA tests came positive, a viral infection seems to be the most probable cause. However, at the time of diagnosis in September 2018, the patient suffered from SLE with a possible exacerbation. She was also strictly complying to the immunosuppressive treatment. It cannot be overlooked that these are recognized as individual, potential sHLH causes.

Establishing the diagnosis of HLH is complicated due to a nonspecific syndrome-like clinical picture and signs and symptoms that often overlap with those of the diseases triggering it [2]. The patients generally present with a high fever and a variety of clinical abnormalities: hepatosplenomegaly, gastrointestinal symptoms, signs of central nervous system involvement, cough and dyspnea, less often rash, and superficial node enlargement $[3,6,9]$. Laboratory diagnostics usually reveal cytopenia in 2 or more cell lines, hyperferritinemia, hypertriglyceridemia, and hypofibrinogenemia. Often present, but much less specific are elevated levels of C-reactive protein, $\mathrm{LDH}$, transaminases and D-dimer, coagulopathy, hyperbilirubinemia, hyponatremia, and hypoalbuminemia [1,2]. Our case illustrates how misleading sHLH presentation could be, in particular in a transplant patient. At the admission, our patient reported only fevers and the physical examination revealed only a single enlarged lymph node. Initial laboratory testing showed cytopenia in all 3 cell lines and renal graft failure. It is of note that pancytopenia in the graft recipients can be caused by immunosuppressants or by CMV infection or the drugs used to treat CMV, that is, the factors that are common in this group of patients [6]. Only when further diagnostics revealed a constellation of severe hyperferritinemia and elevated triglycerides alongside the increased activity of LDH and hypoalbuminemia, a suspicion of hyperinflammatory syndrome could be raised. The final step in establishing the diagnosis was a bone marrow aspiration, which revealed intensive hemophagocytosis (it should be noted that for given specimen histopathological description the changes in blood morphology, although worrisome, were relatively mild). Hemophagocytosis in the

\section{Karger'}


bone marrow is considered to be the hallmark of HLH. Majority of patients with HLH will have a bone marrow biopsy performed due to the cytopenia and often, like in our case, it is the histopathological examination of the specimen revealing hemophagocytosis that suggests HLH. However, it is neither very sensitive nor the specific sign since its prevalence among the patients with HLH varies from 25 to $100 \%$ and may be found in many other inflammatory conditions, such as sepsis, severe infections, and systemic diseases [1, 2, 7].

Both the primary and sHLH can be initially treated using the same protocol introduced in HLH-2004 study. Nevertheless, in SHLH patients, a treatment of the triggering disease should be considered as the top priority $[1,2,6,13]$. In our patient, we had to take into account all of the potential causes. The patient was diagnosed with EBV and CMV infection and was given intravenous ganciclovir. Furthermore, an exacerbation of SLE was suspected, but we decided that the immunosuppressive treatment should not be intensified due to multiple coexisting diseases.

The recommendations for the management of HLH in post-renal transplant patients remain to be specified. It is advised to reduce an immunosuppressive treatment since first, the underlying immunosuppression is a known sHLH risk factor, and second, the majority of sHLH cases in this population has been associated with a viral infection and the reduction of the doses of immunosuppressive drugs may enhance the resistance to the pathogen [6]. The available literature includes a suggestion to stop every immunosuppressive drug with an exception to high-dose steroids [6]. In our case, we decided to continue both everolimus and mycophenolate sodium due to a high risk of transplant rejection in our patient considering her comorbidities, persistent high serum creatinine and urea in follow-up laboratory tests and, later, signs of rejection in graft biopsy. The administration of IVIG is considered a viable treatment escalation option. If patient does not respond to the therapy, etoposide, leukocytopheresis, or plasmapheresis may prove effective according to the anecdotal evidence [6,7]. In our center, we decided to perform PE, and after the first 4 sessions, we saw a large improvement in patient's general condition and laboratory markers. Our patient could benefit from PE in 2 ways: 1 being the suppression of sHLH and the other potentially being removal of autoantibodies connected with SLE - it should not be forgotten that the clinical picture at the time suggested SLE exacerbation. Despite the initial partial recovery, in subsequent months further courses of PE had to be performed with additional administration of IVIG. Mortality rate due to sHLH mortality may reach $30-50 \%$ in the general population but could be much higher in immunocompromised patients such as after organ transplantation [6].

What sets this case apart from other cases with sHLH in post-renal transplant patients is the cavitary lesion in right lung that was discovered while searching for the possible triggering condition. It is suspected that the lung involvement may be present in almost half of patients with HLH $[10,14]$; however, detailed descriptions of the lesions in those patients have been sparse. Radiographs usually portrayed changes like interstitial infiltrates, atelectasis, consolidations, ground-glass opacities, pleural effusions, and mediastinal lymphadenopathies. Some of these findings might have resulted from causes other than HLH itself, such as infections, heart failure, fluid overload, and malignancies [14, 15]. Infiltration of the pulmonary tissues by the histiocytes, although possible, is an extremely rare instance. There was only 1 case report of sHLH in which the intravascular pulmonary histiocytosis was described, manifesting as bilateral pulmonary nodules in a CT scan [10]. Another case report presented a patient with macrophage activation syndrome - a twin-like condition to HLH - with an excavated nodule observed in CT scan and macrophage infiltrates in transbronchial lung biopsy [11].

The cavitary lesion found in our patient did not match any of the typical radiological findings, but it bears some similarities with the excavated nodule in the macrophage activation syndrome patient described by Brandão-Neto et al. [11]. It may also resemble the changes typical for pulmonary fungal infection - it should be noted that pulmonary fungal

\section{Karger'}


Case Reports

in Nephrology

and Dialysis

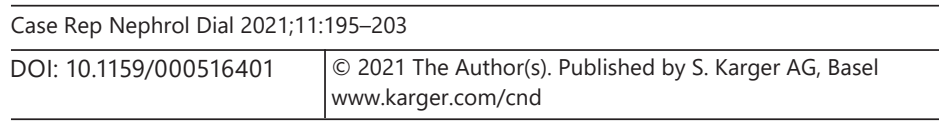

Muras-Szwedziak et al.: sHLH with a Pulmonary Complication in a Kidney Transplant Recipient

infection and sHLH coexistence have already been described $[16,17]$ - but considering the negative fungal cultures, this explanation is unlikely to be correct. Since the cavitary lung lesion in our patient was not present before the sHLH onset, we suspect that it developed as a complication of sHLH. The thoracic surgery and histopathological examination of the specimen would bring a definite answer regarding its etiology. Nevertheless, at the moment of writing this report, it is uncertain whether the benefits from such operation would outweigh the risks. Unless any further enlargement of the lesion or pulmonary complications occur, the potential surgery is being put on hold.

sHLH remains a poorly understood condition, and further research in its diagnostics and treatment is warranted. As reflected by our case, the course of sHLH can be unpredictable and challenging, in particular especially in the immunocompromised post-renal transplant patients. It is important to take sHLH into consideration in the organ transplant recipients presenting with unspecific symptoms accompanied by bicytopenia or sepsis-like clinical picture. When sHLH diagnosis is established, the vast array of laboratory and imaging tests need to be performed in order to identify the underlying condition and potential complications. The treatment should be administered as soon as possible utilizing the protocols that have been proven successful in sHLH patient population.

\section{Statement of Ethics}

Informed written consent was obtained from the patient for being described in this case report and for publication of any accompanying images. The research was conducted ethically in accordance with the World Medical Association Declaration of Helsinki.

\section{Conflict of Interest Statement}

All the authors declare no conflict of interest.

\section{Funding Sources}

The study was financially supported by the Medical University of Lodz grant No. 503/1151-02/503-01.

\section{Author Contributions}

M.T., K.M.-S., and A.M.-Z. contributed equally to the preparation of this manuscript. K.M.-S. and A.M.-Z. were responsible for the management of the patient. M.N. helped in the writing and editing of the original version of the manuscript.

\section{References}

1 Weitzman S. Approach to hemophagocytic syndromes. Hematology Am Soc Hematol Educ Program. 2011; 2011:178.

2 Schram AM, Berliner N. How I treat hemophagocytic lymphohistiocytosis in the adult patient. Blood. 2015; 125(19):2908.

3 Lovisari F, Terzi V, Lippi MG, Brioschi PR, Fumagalli R. Hemophagocytic lymphohistiocytosis complicated by multiorgan failure: acase report. Medicine. 2017;96(50):e9198. 
4 Brisse E, Wouters CH, Andrei G, Matthys P. How viruses contribute to the pathogenesis of hemophagocytic lymphohistiocytosis. Front Immunol. 2017;8:1102.

5 Narciso Júnior J, Neri BO, Dantas GLA, Silveira LHJ, Sales ML, Freitas TVS, et al. Secondary hemophagocytic syndrome after renal transplantation: two case-reports. J Bras Nefrol. 2020;42(1):118.

6 Ponticelli C, Alberighi OD. Haemophagocytic syndrome-a life-threatening complication of renal transplantation. Nephrol Dial Transplant. 2009;24(9):2623.

7 Nusshag C, Morath C, Zeier M, Weigand MA, Merle U, Brenner T. Hemophagocytic lymphohistiocytosis in an adult kidney transplant recipient successfully treated by plasmapheresis: A case report and review of the literature. Medicine. 2017;96(50):e9283.

8 Yang D, Thamcharoen N, Marcus C, Varkaris A, Aird W, Khankin EV, et al. Unusual presentation of hemophagocytic lymphohistiocytosis in a kidney transplant patient. Case Rep Transplant. 2019;2019:3682378.

9 Karras A, Thervet E, Legendre C. Groupe Coopératif de transplantation d'Ile de France. Hemophagocytic syndrome in renal transplant recipients: report of 17 cases and review of literature. Transplantation. 2004; $77(2): 238-243$.

10 Zayed Y, Osman M, Kheiri B, Azher Q, Bachuwa G. Bilateral pulmonary nodules and intravascular pulmonary histiocytosis: a rare presentation of hemophagocytic lymphohistiocytosis secondary to epstein-barr virus infection. Respir Med Case Rep. 2018;26:11.

11 Brandão-Neto RA, Santana AN, Danilovic DL, Bernardi FC, Barbas CS, de Mendonça BB. A very rare cause of dyspnea with a unique presentation on a computed tomography scan of the chest: macrophage activation syndrome. J Bras Pneumol. 2008;34(2):118.

12 Raffray L, Couzi L, Viallard JF, Pellegrin JL, Augis V, Vernant JP, et al. Mycophenolate mofetil: a possible cause of hemophagocytic syndrome following renal transplantation? Am J Transplant. 2010;10(10):2378.

13 Henter JI, Horne A, Aricó M, Egeler RM, Filipovich AH, Imashuku S, et al. HLH-2004: diagnostic and therapeutic guidelines for hemophagocytic lymphohistiocytosis. Pediatr Blood Cancer. 2007;48(2):124.

14 Seguin A, Galicier L, Boutboul D, Lemiale V, Azoulay E. Pulmonary involvement in patients with hemophagocytic lymphohistiocytosis. Chest. 2016;149(5):1294.

15 Fitzgerald NE, MacClain KL. Imaging characteristics of hemophagocytic lymphohistiocytosis. Pediatr Radiol. 2003;33(6):392.

16 Han T, Liu YM, Yang T, Dai HP, Zhang XL. A novel case of pulmonary nocardiosis with secondary hemophagocytic lymphohistiocytosis. Chin Med J. 2017;130(17):2128.

17 Ramsi M, Alvira C, Purohit P, Cornfield D. Haemophagocytic lymphohistiocytosis associated with coccidiomycosis. BMJ Case Rep. 2014;2014:bcr2014205681. 